\title{
Renormalization of the Hartree-Fock-Bogoliubov Equations in the Case of a Zero Range Pairing Interaction
}

\author{
Aurel Bulgac and Yongle Yu \\ Department of Physics, University of Washington, Seattle, WA 98195-1560, USA
}

October 28, 2018

We introduce a natural and simple to implement regularization scheme of the Hartree-Fock-Bogoliubov (HFB) equations with zero range pairing interaction. This renormalization scheme proves to be equivalent to a simple energy cut-off with a position dependent running coupling constant.

PACS numbers: 21.60.Jz, 21.60.-n, 21.30.Fe

More than forty years after the pioneering work of Bohr, Mottelson and Pines [1] there is no need to reiterate again the relevance of pairing correlations in nuclei. It is well established that nuclei are $s$-wave "superconductors" in the so called weak coupling limit, when the pairing gap is much smaller then the fermi energy $\Delta \ll \epsilon_{F}=\hbar^{2} k_{F}^{2} / 2 m$ ( $k_{F}$ is the fermi wave vector). In this limit one can show that the rms radius of the Cooper pair (in infinite matter) significantly exceeds the interparticle separation, $\hbar^{2} k_{F} / m \Delta \gg 1 / k_{F}$ [2], and the radius of the nucleon-nucleon interaction as well. As in the case of the deuteron, the details of the two-particle interaction at distances smaller or comparable with the interaction radius should be irrelevant and the bulk Cooper pair properties should be described basically by a single constant, derivable from a suitably chosen zero range interaction model. (We shall not address here corrections beyond the leading order, such as effective range effects.) One encounters typically no insurmountable difficulties in introducing a local HF (or Kohn-Sham) Hamiltonian $h(\boldsymbol{r})$ 3.,A. If one can adopt the approximation of a zero range two-body interaction in the pairing channel as well then the HFB equations become

$$
\begin{aligned}
& {[h(\boldsymbol{r})-\mu] u_{i}(\boldsymbol{r})+\Delta(\boldsymbol{r}) v_{i}(\boldsymbol{r})=E_{i} u_{i}(\boldsymbol{r}),} \\
& \Delta^{*}(\boldsymbol{r}) u_{i}(\boldsymbol{r})-[h(\boldsymbol{r})-\mu] v_{i}(\boldsymbol{r})=E_{i} v_{i}(\boldsymbol{r}) .
\end{aligned}
$$

Here $u_{i}(\boldsymbol{r})$ and $v_{i}(\boldsymbol{r})$ are the quasi-particle wave functions, $\Delta(\boldsymbol{r})=\frac{\delta E_{g s}}{\delta \nu^{*}(\boldsymbol{r})}$ is the local pairing field, $\mu$ is the chemical potential, $E_{g s}$ is the ground state energy of the system and $\nu(\boldsymbol{r})$ is the anomalous density. In all the formulas presented here we shall not display the spin degrees of freedom. If one takes at face value Eqs. (1),2) one can show that the diagonal part of the anomalous density matrix $\nu(\boldsymbol{r}, \boldsymbol{r})$ diverges, since when $\left|\boldsymbol{r}_{1}-\boldsymbol{r}_{2}\right| \rightarrow 0$ the anomalous density $\nu\left(\boldsymbol{r}_{1}, \boldsymbol{r}_{2}\right)$ has the singular behavior

$$
\nu\left(\boldsymbol{r}_{1}, \boldsymbol{r}_{2}\right)=\sum_{i} v_{i}^{*}\left(\boldsymbol{r}_{1}\right) u_{i}\left(\boldsymbol{r}_{2}\right) \propto \frac{1}{\left|\boldsymbol{r}_{1}-\boldsymbol{r}_{2}\right|},
$$

and the local pairing field $\Delta(\boldsymbol{R})$ cannot be defined [5 7 .

In metals this type of singularity does not play a noticeable role, because the summation over the singleparticle states is cut-off at energies of the order of the Debye energy $\omega_{D} \ll \varepsilon_{F}$. The single-particle density of states is essentially constant in an energy window of width $\mathcal{O}\left(\omega_{D}\right) \ll \epsilon_{F}$ and the expression for the anomalous density has only an infrared logarithmic divergence. This logarithmic divergence is due to states near the Fermi surface and has nothing to do with the ultraviolet divergence due to states faraway from the Fermi surface, which leads to the $1 /\left|\boldsymbol{r}_{1}-\boldsymbol{r}_{2}\right|$ singularity discussed here. The infrared divergence leads to the notorious non-analytical dependence of the gap on the coupling constant, namely $\Delta=\omega_{D} \exp (-1 / V N)$, where $V$ is the strength of the interaction and $N$ is the single-particle density of states at the Fermi energy $\varepsilon_{F}$.

In nuclei and especially in very dilute fermionic atomic systems, where $k_{F} r_{0} \ll 1$ and $r_{0}$ is the radius of the interaction, there is effectively no well defined cut-off and one needs to regularize the theory. A finite range interaction will provide a natural cut-off at single-particle energies of the order of $\varepsilon_{c} \sim \hbar^{2} / m r_{0}^{2}$, when the fast spatial oscillations of the quasi-particle wave functions $u_{i}(\boldsymbol{r}), v_{i}(\boldsymbol{r})$ will render the nonlocal pairing field $\Delta\left(\boldsymbol{r}_{1}, \boldsymbol{r}_{2}\right)$ ineffective. Even though the presence of a finite range of the interaction in the pairing channel formally removes the ultraviolet divergence of the gap, it is very difficult to come to terms with the fact that a cut-off at an energy of the order of $\hbar^{2} / m r_{0}^{2}$ could be the responsible for the definition of the gap both in the case of regular nuclei and very dilute nuclear matter as well. The characteristic depth of the nucleon-nucleon interaction potential, which is of the order of $\hbar^{2} / m r_{0}^{2}$, being the largest energy in the system, can be effectively considered to be infinite in the case of dilute systems. A well defined theoretical scheme for the calculation of a local pairing field, should lead to a converged result when only single-particle states near the Fermi surface are taken into account.

Most of the calculational schemes suggested so far for infinite systems reduce to replacing a zero range potential by a low energy expansion of the vacuum two-body scattering amplitude [2, 15]. The traditional approach in the calculations of finite nuclei consists however in introducing a simple energy cut-off, while the pairing field 
is computed by the means of a pseudo-zero-range interaction. In this approach the effective range of the interaction is obviously determined by the value of the energy cut-off and the two-body coupling constant in the pairing channel is chosen accordingly [16. Such a pure phenomenological approach lacks a solid theoretical underpinning and always leaves the reader with a feeling that "the dirt has been swept under the rug". Another solution favored by other practitioners is to use a finite range two-body interaction from the outset, such as Gogny interaction [17. Besides the fact that the ensuing HFB equations are much more difficult to solve numerically, such an approach also lacks the elegance and transparency of a local treatment and this seemingly simple recipe is indeed as phenomenological in spirit as the treatment based on a pseudo-zero-range interaction, with an explicit energy cut-off. Moreover, in spite of the feeble arguments often put forward in favor of a finite range interaction in $\mathrm{HFB}$ calculations, the only real argument is the fact that the pairing field would otherwise diverge, and there is no mean-field observable which would be noticeable different in the case of a finite range interaction.

The only attempt to implement a consistent regularization scheme for finite systems that we are aware of is that of Ref. [7]. In agreement with the analysis of Ref. [5] the authors of Ref. [7] conclude that in the case of a zero range two-body interaction the anomalous density has a $1 /\left|\boldsymbol{r}_{1}-\boldsymbol{r}_{2}\right|$ singularity. The regularization schemes for infinite homogeneous systems amounts to subtracting a term proportional to $1 / k^{2}$ in the gap equation in momentum representation [2], which in coordinate representation corresponds naturally to a $1 /\left|\boldsymbol{r}_{1}-\boldsymbol{r}_{2}\right|$ term as well. Since the divergence in the anomalous density $\nu\left(\boldsymbol{r}_{1}, \boldsymbol{r}_{2}\right)$ is due to large momenta and thus short distances, it is not surprising that the character of the divergence is not affected by the size of the system. Bruun et al. advocate the use of the following calculational procedure for the anomalous density. First of all one represents the anomalous density as [18]

$$
\begin{aligned}
& \nu\left(\boldsymbol{r}_{1}, \boldsymbol{r}_{2}\right)=\sum_{E_{i}>0}\left[v_{i}^{*}\left(\boldsymbol{r}_{1}\right) u_{i}\left(\boldsymbol{r}_{2}\right)+\frac{\Delta(\boldsymbol{r})}{2} \frac{\psi_{i}^{*}\left(\boldsymbol{r}_{1}\right) \psi_{i}\left(\boldsymbol{r}_{2}\right)}{\mu-\varepsilon_{i}}\right] \\
& -\frac{\Delta(\boldsymbol{r})}{2} G_{0}\left(\boldsymbol{r}_{1}, \boldsymbol{r}_{2}, \mu\right) \\
& {\left[h(\boldsymbol{r})-\varepsilon_{i}\right] \psi_{i}(\boldsymbol{r})=0} \\
& {\left[\mu-h\left(\boldsymbol{r}_{1}\right)\right] G_{0}\left(\boldsymbol{r}_{1}, \boldsymbol{r}_{2}, \mu\right)=\delta\left(\boldsymbol{r}_{1}-\boldsymbol{r}_{2}\right)}
\end{aligned}
$$

where $\boldsymbol{r}=\left(\boldsymbol{r}_{1}+\boldsymbol{r}_{2}\right) / 2$. One can easily justify this subtraction scheme in infinite homogeneous matter, since $v_{i}^{*}\left(\boldsymbol{r}_{1}\right) u_{i}\left(\boldsymbol{r}_{2}\right)=\Delta \psi_{i}^{*}\left(\boldsymbol{r}_{1}\right) \psi_{i}\left(\boldsymbol{r}_{2}\right) / 2 \sqrt{\left(\varepsilon_{i}-\mu\right)^{2}+\Delta^{2}}$. In the limit $\boldsymbol{r}_{1} \rightarrow \boldsymbol{r}_{2}$ the sum over single-particle states in Eq. (4) is converging now and one has only to extract the regulated part of the propagator $G_{0}\left(\boldsymbol{r}_{1}, \boldsymbol{r}_{2}, \mu\right)$, using the pseudo-potential approach [8]

$$
\begin{aligned}
& \nu_{r e g}(\boldsymbol{r}):=\sum_{E_{i}>0}\left[v_{i}^{*}(\boldsymbol{r}) u_{i}(\boldsymbol{r})+\frac{\Delta(\boldsymbol{r}) \psi_{i}^{*}(\boldsymbol{r}) \psi_{i}(\boldsymbol{r})}{2\left(\mu-\varepsilon_{i}\right)}\right] \\
& -\frac{\Delta(\boldsymbol{r})}{2} G_{0}^{r e g}(\boldsymbol{r}, \mu), \\
& G_{0}^{r e g}(\boldsymbol{r}, \mu)=\lim _{\boldsymbol{r}_{1} \rightarrow \boldsymbol{r}_{2}} G_{0}\left(\boldsymbol{r}_{1}, \boldsymbol{r}_{2}, \mu\right)+\frac{m}{2 \pi \hbar^{2}\left|\boldsymbol{r}_{1}-\boldsymbol{r}_{2}\right|}
\end{aligned}
$$

obtaining for the local pairing field

$$
\begin{aligned}
& \Delta(\boldsymbol{r})=\frac{4 \pi|a| \hbar^{2}}{m} \sum_{E_{i}>0}\left[v_{i}^{*}(\boldsymbol{r}) u_{i}(\boldsymbol{r})+\frac{\Delta(\boldsymbol{r}) \psi_{i}^{*}(\boldsymbol{r}) \psi_{i}(\boldsymbol{r})}{2\left(\mu-\varepsilon_{i}\right)}\right] \\
& -\frac{4 \pi|a| \hbar^{2}}{m} \frac{\Delta(\boldsymbol{r})}{2} G_{0}^{r e g}(\boldsymbol{r}, \mu)
\end{aligned}
$$

where $a$ is the two-particle scattering length $(a<0)$. The renormalization procedure and the extraction of the regulated part from various diverging quantities is completely analogous to the familiar procedures in Quantum Field Theory, with the only difference that in this case everything is performed in coordinate space. One literally "throws away" the diverging terms and retains the nonvanishing finite contributions.

The approach suggested in Ref. [7 has however two, related, problems and as is formulated is applicable for systems in a harmonic trap only and does not apply to atomic nuclei or other self-sustaining systems. First of all, after the divergence has been eliminated, the regulated expressions for the anomalous density and for the pairing gap, Eqs. (7,9), are defined entirely in terms of states in a certain neighborhood of the fermi level, since the corresponding sums converge rather quickly. Only when one can establish a one-to-one correspondence between the HFB terms $v_{i}^{*}(\boldsymbol{r}) u_{i}(\boldsymbol{r})$ and the corresponding HF expressions $\Delta(\boldsymbol{r}) \psi_{i}^{*}(\boldsymbol{r}) \psi_{i}(\boldsymbol{r}) / 2\left(\mu-\varepsilon_{i}\right)$ it is clear how to evaluate Eqs. (7,9). There is no one-to-one correspondence for self-sustaining systems [5, 19], where sufficiently deep bound hole states lie in the continuum and where often there is no one-to-one correspondence between the $\mathrm{HF}$ and HFB spectra around the fermi level. In the case of nuclei very close to the nucleon drip lines the HFB spectra are continuous essentially everywhere, while the HF spectra are not, and the one-to-one correspondence between $\mathrm{HFB}$ and $\mathrm{HF}$ is absent. The second and the most difficult aspect of the approach suggested in Ref. [7] however is the fact that it requires the determination of the regular part of the single-particle Green function $G_{0}^{r e g}(\boldsymbol{R}, \mu)$, for which there is so far no clear computational scheme in the case of an arbitrary self-consistent field. These two problems are to a large extent related, as only the whole expressions (79) are uniquely defined, but not each separate part.

Our suggestion amounts to a simple to implement approach. First of all we introduce an explicit energy cutoff $E_{c}$ in evaluating the anomalous density. In this way we can evaluate separately the HFB and HF sums in 
Eqs. (4, 7, 8, 9) irrespective of the existence of the one-toone correspondence discussed above. The final result is independent of $E_{c}$, if this is chosen appropriately. Secondly, we remark that there is no compelling reason to use the exact HF single-particle wave functions, energies and propagator in Eqs. (4, 7, 8, 9) and in order to construct the regulator one can use a Thomas-Fermi approximation for the relevant quantities. Since the divergence has an ultraviolet character, the Thomas-Fermi approximation is particularly well suited [20]. Thus we arrive at the following relations

$$
\begin{aligned}
& G_{0}\left(\boldsymbol{r}_{1}, \boldsymbol{r}_{2}, \mu-U(\boldsymbol{r})\right)=-\frac{m \exp \left(i k_{F}(\boldsymbol{r})\left|\boldsymbol{r}_{1}-\boldsymbol{r}_{2}\right|\right)}{2 \pi \hbar^{2}\left|\boldsymbol{r}_{1}-\boldsymbol{r}_{2}\right|} \\
& =-\frac{m}{2 \pi \hbar^{2}\left|\boldsymbol{r}_{1}-\boldsymbol{r}_{2}\right|}-\frac{i k_{F}(\boldsymbol{r}) m}{2 \pi \hbar^{2}}+\mathcal{O}\left(\left|\boldsymbol{r}_{1}-\boldsymbol{r}_{2}\right|\right), \\
& \nu_{r e g}(\boldsymbol{r}):=\nu_{c}(\boldsymbol{r})+\frac{i \Delta(\boldsymbol{r}) k_{F}(\boldsymbol{r}) m}{4 \pi \hbar^{2}} \\
& +\frac{\Delta(\boldsymbol{r})}{4 \pi^{2}} \int_{0}^{k_{c}(\boldsymbol{r})} k^{2} d k \frac{1}{\mu-\frac{\hbar^{2} k^{2}}{2 m}-U(\boldsymbol{r})+i \gamma} \\
& =\nu_{c}(\boldsymbol{r}) \\
& -\frac{\Delta(\boldsymbol{r}) m k_{c}(\boldsymbol{r})}{2 \pi^{2} \hbar^{2}}\left\{1-\frac{k_{F}(\boldsymbol{r})}{2 k_{c}(\boldsymbol{r})} \ln \frac{k_{c}(\boldsymbol{r})+k_{F}(\boldsymbol{r})}{k_{c}(\boldsymbol{r})-k_{F}(\boldsymbol{r})}\right\}, \\
& \nu_{c}(\boldsymbol{r})=\sum_{E_{i} \leq E_{c}} v_{i}^{*}(\boldsymbol{r}) u_{i}(\boldsymbol{r}), \\
& h(\boldsymbol{r})=-\frac{\hbar^{2} \nabla^{2}}{2 m}+U(\boldsymbol{r}), \\
& E_{c}=\frac{\hbar^{2} k_{c}^{2}(\boldsymbol{r})}{2 m}+U(\boldsymbol{r})-\mu, \\
& \mu=\frac{\hbar^{2} k_{F}^{2}(\boldsymbol{r})}{2 m}+U(\boldsymbol{r}),
\end{aligned}
$$

where the cut-off energy $E_{c}$ is chosen sufficiently far away from the Fermi level to insure that the rhs of Eqs. (11, 12) has converged. As usual one has to take the limit $\gamma \rightarrow 0+$ at the end of the calculations. The local wave vector $k_{F}(\boldsymbol{r})$ is real only in the physically allowed region of the fermi level, where the regularized part of the propagator is imaginary. This imaginary part of the regularized propagator is, naturally, exactly canceled by the corresponding imaginary part of the momentum truncated propagator in Eq. (11). If the Fermi momentum becomes imaginary (outside nuclei for example) one can easily show that $\nu_{r e g}(\boldsymbol{r})$ is still real. The pairing field has thus the simple expression

$$
\begin{aligned}
& \Delta(\boldsymbol{r})=-g_{e f f}(\boldsymbol{r}) \nu_{c}(\boldsymbol{r})=-g \nu_{r e g}(\boldsymbol{r}) \\
& \frac{1}{g_{e f f}(\boldsymbol{r})}=\frac{1}{g} \\
& -\frac{m k_{c}(\boldsymbol{r})}{2 \pi^{2} \hbar^{2}}\left[1-\frac{k_{F}(\boldsymbol{r})}{2 k_{c}(\boldsymbol{r})} \ln \frac{k_{c}(\boldsymbol{r})+k_{F}(\boldsymbol{r})}{k_{c}(\boldsymbol{r})-k_{F}(\boldsymbol{r})},\right] .
\end{aligned}
$$

where $g=4 \pi \hbar^{2} a / m$. Surprisingly, these relations look very much like a simple position or density dependent renormalization of the coupling constant. For a typical nuclear potential which monotonically increases with the radial coordinate $(d U(\boldsymbol{r}) / d r>0)$ one can easily show that $d g_{\text {eff }}(\boldsymbol{r}) / d r>0$, thus the effective pairing interaction is stronger inside than outside nuclear matter (remember $g<0)$. This is stark contrast with the behavior one would get using the popular energy cut-off of a $g \delta\left(\boldsymbol{r}_{1}-\boldsymbol{r}_{2}\right)$ interaction, namely the vacuum renormalization scheme [16]. In this case the effective coupling constant is $g_{v a c}(\boldsymbol{r})=g /\left[1-g m k_{c}(\boldsymbol{r}) / 2 \pi^{2} \hbar^{2}\right]$ and one can then easily show that $d g_{v a c}(\boldsymbol{r}) / d r<0$ if $d U(\boldsymbol{r}) / d r<0$.

It is instructive to apply this recipe to the case of infinite homogeneous matter. After a few simple manipulations one can show that the equation for the gap reads

$$
\begin{aligned}
& \frac{1}{k_{F}} \int_{0}^{k_{c}} d k \frac{k^{2}}{\sqrt{\left(k^{2}-k_{F}^{2}\right)^{2}+k_{P}^{4}}} \\
& =\frac{\pi}{2 k_{F}|a|}\left[1+\frac{2 k_{c}|a|}{\pi}-\frac{k_{F}|a|}{\pi} \ln \frac{k_{c}+k_{F}}{k_{c}-k_{F}}\right],
\end{aligned}
$$

where $k_{P}^{2}=2 m \Delta / \hbar^{2}$. Using the methods described in Refs. [2.913] one would not get the term with the $\log -$ function. The technical reason is that we used $\Delta /\left(\varepsilon_{i}-\mu\right)$ instead of $\Delta / \varepsilon_{i}$ in Eqs. (4,7, 9, 11]) respectively [21], which enhances the convergence of the corresponding sums or integrals discussed above. Parametrically we are allowed to make such a substitution as long as $\left|k_{F} a\right| \ll 1$, otherwise one should consider effective range corrections and higher partial waves. Even though the momentum cutoff $k_{c}$ appears explicitly here, once this momentum cutoff is sufficiently large, there is no dependence of the gap on the cut-off momentum.

When evaluating the total energy of the system one has to be careful and calculate the expression [12]

$$
E_{g s}=\int d^{3} r\left[\frac{\hbar^{2}}{2 m} \tau_{c}(\boldsymbol{r})-\Delta(\boldsymbol{r}) \nu_{c}(\boldsymbol{r})\right]+E_{p o t},
$$

where $E_{p o t}$ is the usual HF potential energy contribution, since the kinetic energy density $\tau_{c}(\boldsymbol{r})=$ $2 \sum_{E \leq E_{c}}\left|\nabla v_{E}(\boldsymbol{r})\right|^{2}$ diverges in a similar fashion as $\nu_{c}(\boldsymbol{r})$ $E_{c}$, but $E_{g s}$ does not.

We have implemented this renormalization scheme for the pairing field for both selfconsistent and nonselfconsistent calculations of spherical nuclei. The normal and anomalous densities were computed following the complex energy integration technique extensively used by Fayans and his collaborators [15]. In order to illustrate the convergence properties we present in Fig. 1 the neutron pairing field $\Delta(\boldsymbol{r})$ obtained as a solution of the Eqs. (1, 2, 17, 18) for a range of cut-off energies $E_{c}$. The calculations were performed for a simple WoodsSaxon potential with fixed parameters corresponding to a ${ }^{110} \mathrm{Sn}$ nucleus [22] and for a fixed value of the chemical potential $\mu=-0.1 \mathrm{MeV}$ (essentially at the neutron drip line). The value of the bare coupling constant is 
$g=-200 \mathrm{MeV} \cdot \mathrm{fm}^{3}$. The total energy converges equally fast with $E_{c}$. The reasons why convergence is achieved for $E_{c}=\mathcal{O}\left(\epsilon_{F}\right)$ and how one can improve on this aspect are discussed in Ref. 23.].

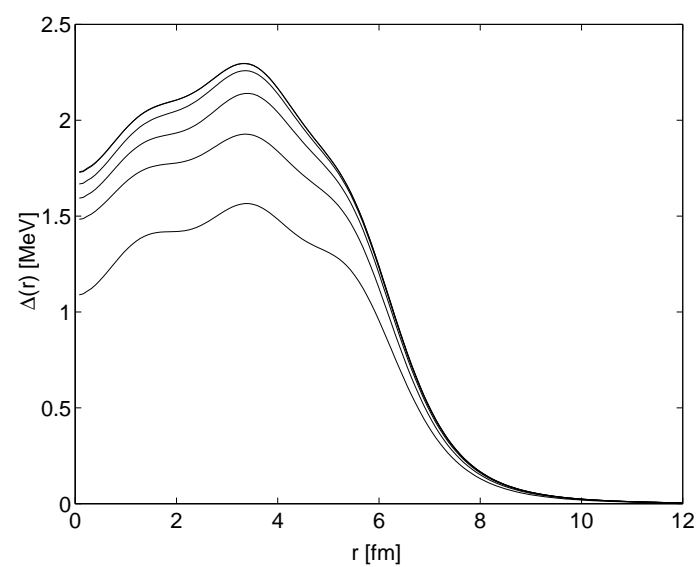

FIG. 1. The neutron pairing field $(17)$ as a function of the radial coordinate and of the cut-off energy $E_{c}$. Upward various curves correspond to $E_{c}=20,30,35,40,45$ and $50 \mathrm{MeV}$ respectively. On the scale of the figure the last two curves are indistinguishable.

In conclusion, we have presented a renormalization procedure for the HFB equations in the case of zero range pairing interaction, which is easy to implement for any type of finite or infinite systems and which converges very fast as well. A very interesting feature of this approach is its similarity with a density dependence of the pairing interaction. The numerical implementation of the present renormalization scheme is straightforward and amounts to very small changes of the existing codes.

We thank DoE for financial support, G.F. Bertsch and J. Dobaczewski for discussions and G. Bruun for drawing our attention to our initial misinterpretation of the symbol $E_{\eta}^{0}$ in Eq. (27) of Ref. [7]. The very warm hospitality of N. Takigawa in Sendai and the financial support of JSPS were very helpful while AB was writing the final version of this work.

[1] A. Bohr, B.R. Mottelson and D.Pines, Phys. Rev. 110, 936 (1958).

[2] M. Randeria, in Bose-Einstein Condensation, eds. A. Griffin, D.W. Snoke and S. Stringari, Cambridge University Press (1995), pp 355-392.

[3] P. Hohenberg and W. Kohn, Phys. Rev. 136, B864 (1964); W. Kohn and L. J. Sham, Phys. Rev. 140, A1133 (1965); R.M. Dreizler and E.K.U. Gross, Density Func- tional Theory: An Approach to the Quantum Many-Body Problem, (Springer, Berlin, 1990).

[4] J.W. Negele and D. Vautherin, Phys. Rev. C 5, 1472 (1972); A. Bulgac, C. Lewenkopf and V. Mickrjukov, Phys. Rev. B 52, 16476 (1995).

[5] A. Bulgac, preprint FT-194-1980, CIP, Bucharest; nuclth/9907088.

[6] The notations used in Ref. [- 5 are slightly different from those commonly used in literature, which we try to follow in this work.

[7] G. Bruun, Y. Castin, R. Dum and K. Burnett, Eur. Phys. J. D 7, 433 (1999).

[8] J.M. Blatt and V.F. Weiskopf, Theoretical Nuclear Physics, Wiley, New York (1952), pp. 74-76; K. Huang, Statistical Mechanics, John Wiley \& Sons, New York (1987), pp 230-238. The procedure amounts to the replacement of the short range potential $V(\boldsymbol{r})$ according to the simple prescription $V(\boldsymbol{r}) \psi(\boldsymbol{r}) \rightarrow g \delta(\boldsymbol{r}) \partial_{r}[r \psi(\boldsymbol{r})]$, where the coupling constant is determined by the scattering length $g=4 \pi a \hbar^{2} / \mathrm{m}$. One can include corrections to the leading order term of the pseudo-potential by making the replacement $a \rightarrow-f /(1+i k f)$, where $f$ is the $s$-wave scattering amplitude. If needed, one can also include higher partial waves 9 .

[9] K. Huang and C.N. Yang, Phys. Rev. 105, 767 (1957); T.D. Lee and C.N. Yang, Phys. Rev. 105, 1119, (1957).

[10] A.A. Abrikosov, A.P. Gorkov and I.E. Djaloshinski, Methods of Quantum Field Theory in Statistical Physics, Dover, New York (1975), Ch. 1.5.

[11] M. Randeria, J.-M. Duan, L.-Y. Shieh, Phys. Rev. B 41, 327 (1990); C.A.R. Sá de Melo, M. Randeria, and J.R. Engelbrecht, Phys. Rev. Lett. 71, 3202 (1993).

[12] T. Papenbrock and G.F. Bertsch, Phys. Rev. C 59, 2052 (1999).

[13] S.D.H. Hsu and J. Hormuzdiar, nucl-th/981101.

[14] V.A. Khodel, V.V. Khodel and J.W. Clark, Nucl. Phys. A 598, 390 (1996).

[15] S. Fayans, JETP Lett. 70, 240 (1999); S.A. Fayans et al., Nucl. Phys. A 676, 49 (2000).

[16] H. Esbensen, G.F. Bertsch and K. Hencken, Phys. Rev. C 56, 3054 (1997).

[17] P. Ring and P. Schuck, The Nuclear Many-Body Problem, Springer, New York (1980), Ch. 4.

[18] The definition of the propagator used in Ref. [7] differs by a sign from the commonly accepted one.

[19] J. Dobaczewski, H. Flocard and J. Treiner, Nucl. Phys. A 422, 103 (1984).

[20] Since the Thomas-Fermi approximation is used for estimating the regulator and not the anomalous density or the pairing gap, no observables are affected.

[21] The authors of Ref. [7] state that previous authors, in particular those of Ref. [11], have used $1 /\left(\varepsilon_{i}-\mu\right)$ and not $1 / \varepsilon_{i}$ as a subtraction scheme. Such a replacement is accurate up to effective range corrections only.

[22] A. Bohr and B. Mottelson, Nuclear Structure, vol. I. (Benjamin, New York, Amsterdam, 1974).

[23] A. Bulgac, nucl-th/0108014. 